wavelength region', J. Lightwave Technol, 1988, LT-8, (2), pp. 273-280

3 KAVEHRAD, M., and sAVov, E. 'Fiber-optic transmission of microwave 64QAM signals', IEEE J. Sel. Areas Commun., 1990, SAC-8, (7), pp. 1320-1325

4 DARCR, T. E.: 'Subcarrier multiplexing for lightwave networks and video distribution systems', IEEE J. Sel. Areas Commun., 1990, SAC-8, (7), pp. 1240-1248

5 POschiN, G. J., and SALEH, A. M.: 'Overcoming optical amplifier intermodulation distortion using coding in multichannel communications systems', IEEE Trans., 1990, COM-38, (2), pp. 187-191

6 OHTSUKA, H., KAGAMI, O., KOMAKI, S., KOHYYMA, K., and KAVEHRAD, M.: '256-QAM subcarrier transmission using coding and optical intensity modulation in distribution networks', IEEE Photonics Technol. Lett., 1991, 3, (4), pp. 381-383

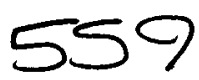

\section{ENERGY QUANTISATION IN FIGURE EIGHT FIBRE LASER}

\section{A. B. Grudinin, D. J. Richardson and D. N. Payne}

Indexing terms: Lasers and laser applications, Nonlinear optics

For the first time the observation of discrete jumps in the power output of an all-fibre passively mode-locked laser is reported. The discontinuities are associated with quantisation of the energy of the pulses circulating within the laser cavity.

Introduction: The passive generation of femtosecond soliton pulses in erbium-doped fibre lasers has recently become of significant interest [1-3]. The figure eight laser configuration [2-4] whose operation is based on the reflection properties of the nonlinear amplifying loop mirror (NALM) [5] has been the object of considerable study and has so far been shown capable of generating bandwidth-limited pulses as short as $320 \mathrm{fs}[2,3]$. In this scheme the quantisation of the soliton energy and the random nature of the pulse formation lead to several distinct regimes of pulse repetition rate behaviour [4]. In general, within one round trip period the solitons are randomly separated in time and the pulse trains repeat at the cavity round trip period. The exact pulse pattern and the total number of pulses in the cavity are highly sensitive to the input pump power. The repetition rate can be stabilised by incorporating a pulse multiplier into the cavity [6]; however, although the general principle of operation of the figure eight laser is understood, many features of its behaviour still remain to be investigated and explained.

We present for the first time experimental results showing the effects of pulse energy quantisation in the power output from the laser. The results demonstrate the preference of the laser to operate in well defined soliton units and its ability to adapt itself to changes of input pump power once soliton selfstarting has occurred.

Experiment: The experimental setup is shown in Fig. 1. The laser was configured to generate transform-limited, $450 \mathrm{fs}$

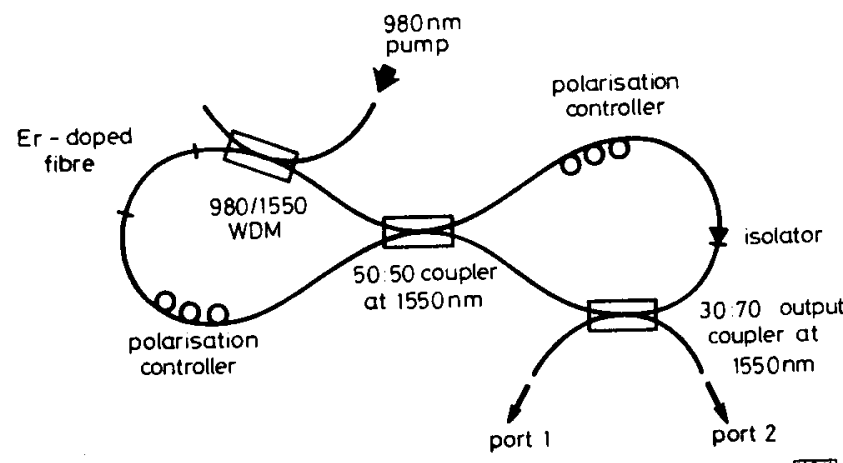

Fig. 1 Experimental configuration soliton pulses at $1558 \mathrm{~nm}$. The NALM loop was $35 \mathrm{~m}$ long and the cavity round trip frequency was $5 \mathrm{MHz}$. The amplifier section consisted of $3 \mathrm{~m}$ of $800 \mathrm{ppm}$ erbium-doped fibre and was pumped by a $\mathrm{Ti}$ : sapphire laser operating at $980 \mathrm{~nm}$. An acousto-optic modulator was placed in front of the $980 \mathrm{~nm}$ pump launch stabilisation circuitry, the $980 \mathrm{~nm}$ pump level to we were able to lock the input pump power to the figure-eight laser to an externally defined reference voltage. Thus by applying a slow sawtooth waveform (100s period) to the pump launch stabilisation circuitry the $980 \mathrm{~nm}$ pump level to the laser could be controllably and repeatedly varied, while measuring the average output power at port 1 on a slow detector (light emanating at port 1 has been switched by the NALM and is the correct port for soliton output). The pulse repetition rate behaviour and optical spectra were simultanously measured at port 1 . The same measurements could also be made at port 2 where light reflected by the NALM (i.e. the nonsolitonic component) can be observed.

A typical laser output characteristic measured at port 1 is shown in Fig. 2 and exhibits a number of interesting features, such as discrete jumps and hysteresis in the output power. The $\mathrm{CW}$ lasing threshold is seen to occur at approximately $10 \mathrm{~mW}$, whereas a 'second threshold' occurs at around $45 \mathrm{~mW}$, where soliton modelocked operation selfstarts. Once pulsed operation initiates it can be sustained at reduced pump power levels, down to within a few milliwatts of the CW laser threshold, giving rise to a hysteresis in the power output.

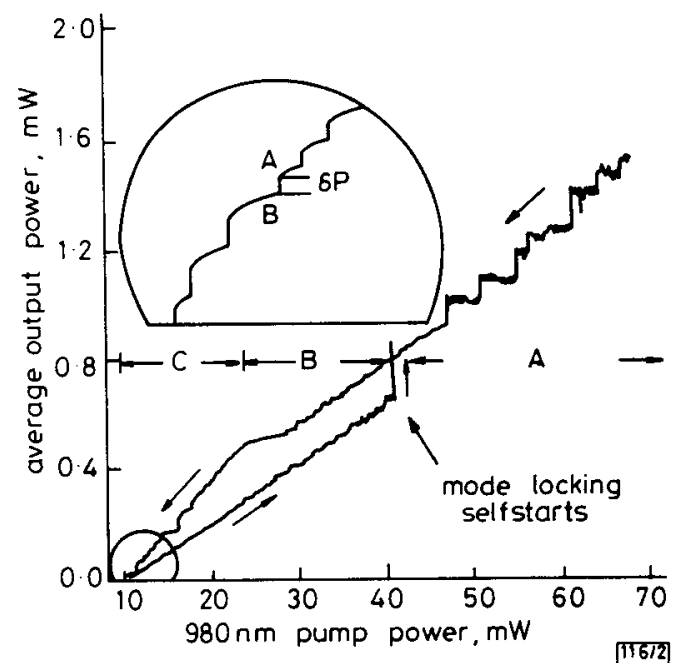

Fig. 2 Typical laser characteristic

Inset: output power, on an expanded scale, at low values of input pump power (Note: inset laser characteristic corresponds to another hysteresis measurement from small scale curve)

In the time domain, three regions of pulsed operation can be identified, corresponding to regions $\mathrm{A}-\mathrm{C}$ on the laser output characteristic (Fig. 2).

(i) Region A: For pump powers above the mode-locking selfstart threshold, many pulses exist within the laser resonator. The pulses undergo complicated motions with respect to each other, possibly due to soliton-soliton interactions. Owing to the random nature of the laser pulsing, the pulse patterns and motions vary with time and the detailed features of the laser characteristic similarly differ for each sweep of the pump power. A small CW component is also apparent.

(ii) Region $B$ : As the pump power is lowered below the selfstarting threshold the pulse motions become progressively less chaotic and the laser output smoother until, at a power level of $25 \mathrm{~mW}$, the output power curve displays a distinct hump.

(iii) Region $C$ : The laser slope efficiency is increased below the output characteristic hump. In the time domain the pulses group themselves into ordered patterns and all motions cease (see Fig. 3). The small accompanying CW level at the output noted at higher pump powers also disappears. As the pump power is further reduced abrupt jumps (inset Fig. 2) in output power are observed and are associated with the disappearance 
of individual pulses from the pulse trains. The effect is illustrated in Fig. 3 where the pulse train is shown before and after a power jump (points $A$ and $B$, inset Fig. 2). Fig. 3 clearly shows that the number of pulses circulating in the cavity drops from 6 to 5 at the power discontinuity. Pulses can vanish from the trains singly or a number of pulses $(n=2,3,4$ ...) can disappear simultaneously (with a correspondingly larger drop in average output power). Measurements at port 2 show an abrupt increase in average output power on the disappearance of a soliton. Region $\mathrm{C}$ is the most interesting and controllable and the remainder of this paper deals with it exclusively.

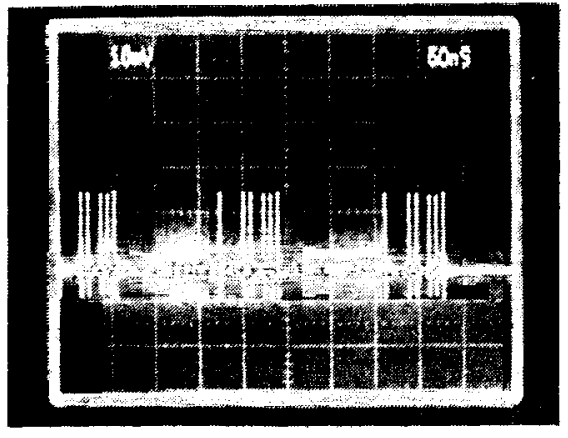

a

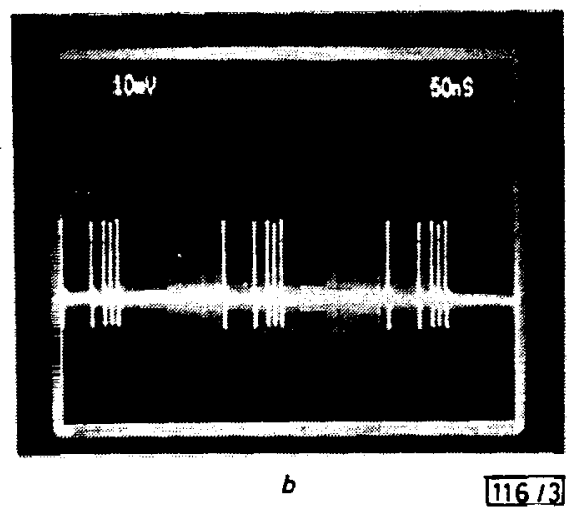

Fig. 3 Temporal behaviour of laser output

$a$ At point A on inset Fig. 2
$b$ At point B on inset Fig. 2

By measuring the change in output power $\delta \mathrm{P}=13 \mu \mathrm{W}$ (Fig. 2), we can estimate the energy of the pulses circulating within the unidirectional loop of the laser. The calculated energy $E=12 \mathrm{pJ}$ indicates that the pulses of duration $450 \mathrm{fs}$, circulating within the isolator loop have a soliton number in the range $1.0<N<1.4$ (fibre dispersion $=3.5 \mathrm{ps} / \mathrm{nm} \mathrm{km}$ ). Optical spectra measurements at points before and after a power discontinuity reveal the appearance of additional, low level, symmetric spectral lobes on the otherwise smooth intensity spectrum at port 1 . This is accompanied by the enhancement of the central wavelength component at port 2 .

Discussion: The measurements are consistent with the postulate that, because of the presence of the NALM within the laser resonator, a soliton pulse experiences the lowest loss on circulation around the cavity and the laser is therefore predisposed towards operating with solitons within the cavity. Owing to intensity changes around the fibre circuit the pulse is not a perfect soliton at all points within the cavity. Nonsoliton components generated within a round trip are however rejected by the NALM and are lost within the isolator. The number of pulses within the cavity is therefore quantised and determined by the pump power level. A reduction in pump power can only reduce the number of pulses by one (or an integer number) of pulses and this leads to (quantised) step discontinuities in the laser output power. Any circulating power excess above that required to support this new number of solitons is rejected from the system by the combined operation of the switch, which preferentially transmits the soliton pulse component, and the isolator.
In reality it is found that the soliton puise energy as observed at port 1 is not precisely quantised and varies by about $5 \%$ between jumps in the number of circulating pulses; this allows for the smooth decrease in output power between jumps (inset Fig. 2). As expected, however, on dropping a pulse from the cavity, a large scale $(30 \%)$ variation in the output intensity at port 2 (the NALM rejection port) is observed as the system adjusts to the new, reduced, circulating power. Further measurements indicate that the soliton pulses within groups exhibit a small pulse to pulse energy variation at port 1 , of the order of a few percent. This variation, possibly due to very slight differences in polarisation states or central frequencies of the individual pulses, may facilitate the removal of a particular pulse (or a group of pulses) from the cavity as the amplifier pump power is reduced.

Conclusions: Our results demonstrate energy quantisation effects in the figure eight laser and show the mechanism by which the system is able to adapt to continuous changes in pump power. In addition, we have shown that circulating pulses within the cavity are close to being fundamental solitons. The challenge remains to control the pulse patterns in a predetermined way to generate, high bit rate, soliton sequences.

22nd October 1991

A. B. Grudinin*, D. J. Richardson and D. N. Payne (Optoelectronics Research Centre, Southampton University, Southampton SO9 $5 \mathrm{NH}$ United Kingdom)

- On leave from: Optical Fibre Department, General Physics Institute, Moscow, Russia

\section{References}

1 ZIRNGiBl, M., STULZ, L. W., STONE, J., HUGI, J., DIGIOVANNI, D., and HANSEN, P. B.: '1.2 ps pulses from passively mode-locked, diode pumped Er doped ring laser', Electron. Lett., 1991, 27, pp. 17341735

2 DULING III, I.: 'Sub picosecond all-fibre erbium laser', Electron. Lett., 1991, 27, pp. 544-545

3 RICHARDSON, D. J., LAMING, R. I., PAYNE, D. N., PHILLIPS, M. W., and MATSAS, v.: 'Self-starting passively mode-locked erbium-doped laser', Electron. Lett., 1991, 27, pp. 542-544

4 RICHARDSON, D. J., LAMING, R. I., PAYNE, D. N., PHILliPS, M. W., and MATSAS, v.: 'Pulse repetition rates in passive, selfstarting, femtosecond soliton fibre laser', Electron. Lett., 1991, 27, pp. 1451-1453

5 RICHARDSON, D. J., LAMING, R. 1., and PAYNE, D. N.: 'Ultra low threshold Sagnac switch incorporating an EDFA', Electron. Lett., 1990,26 , pp. 1779-1781

6 NAKAZAWA, M., YoshiDA, E., and KimURA, Y.: 'Repetition rate control of an LD pumped femtosecond erbium doped fibre laser using a sub ring cavity'. Nonlinear Guided Wave Phenomena, 1991, PDP-9

\section{ENHANCEMENT OF EFFECTIVE SCHOTTKY BARRIER HEIGHT ON $\boldsymbol{n}$-TYPE InP}

\author{
M. C. Ho, Y. He, T. P. Chin, B. W. Liang and \\ C. W. Tu
}

Indexing terms: Schottky contacts, Semiconductor growth, Semiconductor devices and materials

The effective Schottky barrier height on n-type InP is increased by a thin heavily-doped p-type surface layer grown by gas-source molecular beam epitaxy. The relationships between the barrier height increment and the doping level and thickness of the surface layer have been studied. The Schottky diodes fabricated by this method show reasonably low leakage current at high reverse bias and high reverse break-down roltage.

Introduction: InP is an important material for high-speed electronic and optoelectronic application $[1,2]$, but it has a major disadvantage of low Schottky barrier height [3]. This inher- 